\title{
Cell Sheets for Periodontal Tissue Engineering
}

\author{
Takanori Iwata $^{1,2} \cdot$ Masayuki Yamato $^{1}$ Kaoru Washio ${ }^{1}$ Tomohiro Ando ${ }^{2}$. \\ Teruo Okano ${ }^{1} \cdot$ Isao Ishikawa $^{1}$
}

Published online: 10 September 2015

(C) Springer International Publishing AG 2015

\begin{abstract}
Cell Sheet Technology was first utilized in a clinical trial for corneal reconstruction in 2003; since then, several clinical trials have been performed not only in Japan but also in Europe. The key technology of Cell Sheet is a temperatureresponsive polymer (poly $N$-isopropylacrylamide), which characteristics dramatically change from hydrophobic to hydrophilic by changing the temperature. This intelligent polymer is covalently grafted onto the surface of cell culture dishes, where cells can attach and proliferate at $37^{\circ} \mathrm{C}$. Reducing the temperature below $32{ }^{\circ} \mathrm{C}$ causes this surface to hydrate; therefore, confluent cells detach from the surface as a sheet. Cell sheets can be harvested without the use of typical proteolytic enzymes; thus, intact cell-cell interaction, cell surface proteins, and extracellular matrix proteins are preserved.
\end{abstract}

This article is part of the Topical Collection on Stem-Cell Biology for Tooth and Periodontal Regeneration

Takanori Iwata

iwata.takanori@twmu.ac.jp

Masayuki Yamato

masayuki.yamato@twmu.ac.jp

Kaoru Washio

washio.kaoru@twmu.ac.jp

Tomohiro Ando

tando@oms.twmu.ac.jp

Teruo Okano

tokano@twmu.ac.jp

Isao Ishikawa

ishikawa.isao@twmu.ac.jp

1 Institute of Advanced Biomedical Engineering and Science, Tokyo Women's Medical University, 8-1 Kawada-cho, Shinjuku-ku, Tokyo 162-8666, Japan

2 Department of Oral and Maxillofacial Surgery, Tokyo Women's Medical University, 8-1 Kawada-cho, Shinjuku-ku, Tokyo 162-8666, Japan
Using this technology, we started a clinical trial in 2011 to test the safety and efficacy of autologous periodontal ligament cell sheets for periodontal regeneration.

Keywords Cell sheet · Temperature-responsive polymer . Periodontal ligament cells $\cdot$ Translational research $\cdot$ Clinical trial

\section{Introduction}

Periodontitis affects patients' quality of life; therefore, various regenerative therapies have been attempted since the early twentieth century. Several procedures, such as bone grafts, barrier membranes, and cytokine therapies, are performed in clinics; however, it is difficult to regenerate periodontal tissue completely. Especially for large defects, there is no standard therapy to predictably regenerate damaged periodontal tissue. Thus, cytotherapeutic approaches have been introduced based on the concept of "Tissue Engineering" proposed by Langer and Vacanti [1]. Researchers have now commenced studies to regenerate periodontal tissue using scaffolds, growth factors, and cells based on this concept [2].

\section{Cell Sheet Transplantation as a Scaffold-Free Transplantation}

Our laboratory has studied a "smart material" which is a temperature-responsive polymer named poly $(N$ isopropylacrylamide), and its characteristics dynamically change by simple temperature change. Poly $(N-$ isopropylacrylamide) is covalently attached onto the surface of cell culture dish by electron beam irradiation [3], and the thickness is controlled to approximately $20 \mathrm{~nm}$. Poly $(N$ - 
isopropylacrylamide) is a nanocoating material, and its toxicity for cell is negligible. This cell culture surface is slightly hydrophobic under cell culture conditions at $37{ }^{\circ} \mathrm{C}$ but dramatically changes its characteristics to hydrophilic below its lower critical solution temperature (LCST), $32{ }^{\circ} \mathrm{C}$. Thus, it is possible to control the attachment and detachment of cells by simple temperature change. On this smart surface, cells can spread and grow in a way similar to that seen on untreated normal cell culture surfaces at $37{ }^{\circ} \mathrm{C}$. Furthermore, cells can be detached by reducing temperature below the LCST, allowing for cell harvest from the culture surfaces. The application of this technology enables retrieval of cells as a sheet without proteolytic enzyme treatment, such as trypsin. Therefore, cell-cell interactions, cell surface proteins, and extracellular matrix proteins are preserved within a sheet-like structure. In addition, cell sheets contain intact cell adhesion molecules, which are working as a natural glue; thus, we can transplant cell sheets without the need for sutures. This technology has been applied in clinical trials, such as corneal reconstruction $[4,5]$, esophageal regeneration after endoscopic submucosal dissection [6], cardiac regeneration [7], and cartilage regeneration [8] in Japan and Europe. In 2011, we commenced a clinical trial named "Periodontal regeneration with autologous periodontal ligament cell sheets." In this paper, we describe the translational research of periodontal ligament (PDL) cell sheets and how to create PDL cell sheets.

Before a clinical trial commences, researchers must confirm the safety and efficacy of the materials under investigation with animal studies. In the periodontal field, rats are often used as the first step, because mice are too small to create surgical periodontal defects. Large animals such as dogs are commonly used because their tooth size is similar to human. Several kinds of defect models have been established, and comparative analysis with historical studies can be performed [9]. Recently, some studies have shown that minipigs are a suitable alternative to evaluate the efficacy of cell transplantation $[10,11]$.

The first transplantation of PDL cell sheets was performed by Hasegawa et al. and published in 2005. Human PDL (hPDL) cell sheets were successfully created and transplanted to the periodontal defects of nude rats (athymic rats) xenogenically [12]. In this study, the cell outgrowth method was utilized to obtain the primary culture of hPDL cells. The hPDL cell sheets cultured with ascorbic acid were harvested from temperature-responsive culture dishes and transplanted as cell pellets into a mesial dehiscence model in athymic rats. Four weeks after the transplantation, newly formed PDL-like structures were observed in all the experimental sites. In contrast, such findings were not observed at the control sites. These results suggested that PDL cell sheets contribute to the regeneration of PDL. Next, the optimal culture condition of hPDL cells was investigated. An in vitro study demonstrated that the osteoinductive medium, which consists of ascorbic acid, $\beta$-glycerophosphate, and dexamethasone, enhanced both osteoblastic and periodontal differentiation of hPDL cells [13]. Therefore, hPDL cell sheets cultured with or without these osteoinductive supplements were compared in a rat xenogeneic transplantation model [14]. The three-layered hPDL cell sheets-dentin block constructs induced cementum-like hard tissue on the surface of the dentin in most cases. Welloriented collagen fibers, similar to native Sharpey's fibers, were inserted vertically into the newly formed cementumlike tissue [15]. These results suggested that hPDL cell sheets cultured with osteoinductive medium contribute to the regeneration of both cementum and PDL. From these studies in rats, we could not observed any bone regeneration; thus, we thought that bone filler materials should be needed to regenerate periodontal tissue.

Based on the successful results from studies in rats, we progressed to canine periodontal defect models, because canine models are commonly used to evaluate the safety and efficacy of biomaterials, and the size of their teeth is similar to the human clinical situation [16]. From this study, we introduced collagenase/dispase digestion [17] to dissociate PDL tissue. Dog PDL (dPDL) cells were cultured with osteoinductive supplements for 5 days. Longer periods of cultivation resulted in spontaneous dPDL cell sheet detachment from the temperature-responsive culture surfaces. Similar spontaneous detachment of PDL cell sheets was also observed for porcine cells. The detachment of cells may be due to the tension of extracellular matrix proteins, such as type I collagen, because spontaneous detachment is hardly observed when cells are cultured without ascorbic acid. Three-layered dPDL cell sheets were stacking with woven polyglycolic acid (PGA) $\left(\right.$ Neoveil $^{\circledR}$, PGA Felt-Sheet Type, $0.15 \mathrm{~mm}$ in thickness: Gunze, Tokyo, Japan) and transplanted into the surface of dental roots containing three-wall periodontal defects autologously, and bone defects were filled with porous betatricalcium phosphate ( $\beta$-TCP). Autologous dPDL cell sheets combined with $\beta$-TCP resulted in regeneration of periodontal tissue almost completely in this canine three-wall periodontal defect model. On the other hand, limited bone regeneration without well-oriented PDL was observed in the control group ( $\beta$-TCP only), suggesting that PDL cell sheets with $\beta$-TCP can contribute to complete regeneration of periodontal tissues [13].

Next, we tried a one-wall infrabony defect model [18], which is a more severe defect model than three-wall model [19]. In this study, we evaluated the differences of cell sources for periodontal regeneration. PDL cells, iliac bone marrowderived mesenchymal stromal cells, and alveolar periostealderived cells were obtained from each dog, respectively. Then, three-layered cell sheets were transplanted autologously, and bone defects were filled with porous $\beta$-TCP, which was mixed with $3 \%$ type I collagen gel (Koken, Tokyo, Japan) to stabilize the granule structure. Eight weeks after the 
transplantation, well-oriented PDL fibers and significant newly formed cementum were observed in the PDL cell sheet group only. These results also suggested that PDL-derived cells are the most suitable cell source for periodontal regeneration and that PDL cell sheets with $\beta-\mathrm{TCP} /$ collagen serve as a promising tool for large periodontal defects.

Establishment of a culture method for hPDL cells obtained from a single tooth is essential in performing autologous PDL cell sheet transplantation. However, optimal methods for the isolation and expansion of hPDL cells from a single tooth had not been well investigated. Therefore, various experiments were performed to determine optimal isolation and expansion of hPDL cells using 41 teeth [20]. The hPDL cells were successfully digested with collagenase/dispase and cultured at low cell density to allow clonal proliferation and enrichment of stem cells. Typically, only 10 to 100 colonies of hPDL cells were obtained from a single tooth. The cells were subcultured twice a week to keep a low cell density, and the number of cells usually exceeded one million within 2 weeks. The cells were then switched to the temperature-responsive dishes to create cell sheets in the presence of the osteoinductive supplement. hPDL cell sheets were harvested 2 weeks after spreading for subsequent induction of osteoblastic/periodontal differentiation. Safety tests and quality control tests were performed before the transplantation.

According to the good clinical practice (GCP) and good manufacture practice (GMP) guidelines, hPDL cell sheets were produced at the GMP-grade Cell Processing Center (CPC) in our university [21] (Fig. 1). Certified reagents and materials were selected, and "the product" (PDL cell sheets in

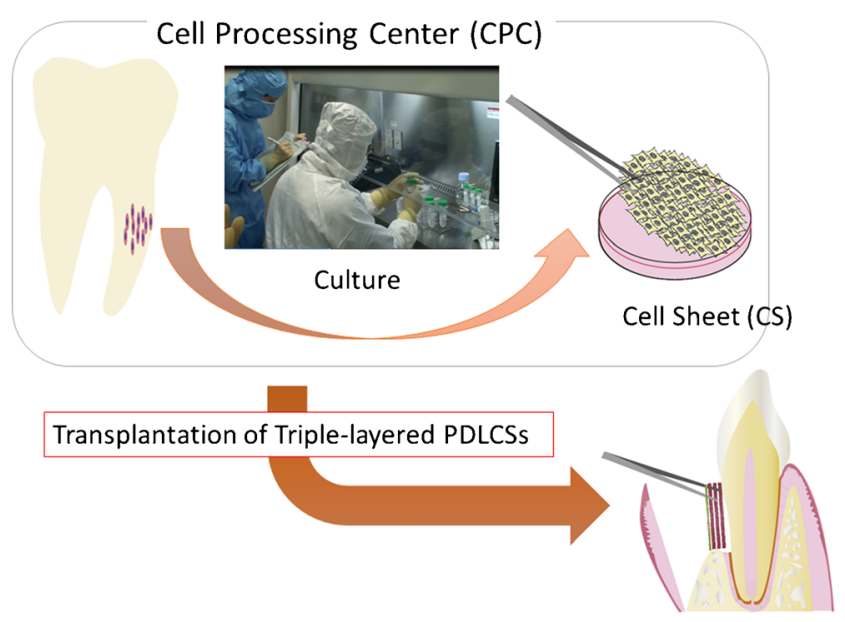

Fig. 1 The procedure of clinical trial titled "Periodontal Regeneration with Autologous Periodontal Ligament Cell Sheets." PDL cells are processed in Cell Processing Center (CPC) of our university, where a clean environment is validated. Patient's PDL cells are proliferated with autologous serum for 2 weeks; then, the cells are spread on the temperature-responsive culture dishes and cultured with osteoinductive medium for additional 2 weeks. Triple-layered PDL cell sheets are constructed and transplanted to the root, and bony defects are filled with porous beta-tricalcium phosphate $(\beta-\mathrm{TCP})$ this case) was produced to test their safety and efficacy. hPDL cells were cultured with autologous serum, and cell sheets were constructed using the temperature-responsive dishes in the CPC. Quality control tests were performed using conventional tests. The safety of the products was investigated using the following tests: (1) soft agar colony formation assay, (2) transplantation into nude mice, and (3) the karyotype test [22]. The safety and efficacy of the cell sheets were assayed by xenogenic transplantation into SCID mice. All of these tests confirmed that hPDL cell sheets created in the CPC were safe and effective for periodontal regeneration.

According to the guidelines of both GCP and GMP, we prepared documents detailing the protocol and submitted it to the Ministry of Health, Labour and Welfare in Japan. Our clinical trial named Periodontal regeneration with autologous periodontal ligament cell sheets was approved and commenced in January 2011. Inclusion criteria included the following: (1) infrabony defects with a probing depth of more than $4 \mathrm{~mm}$ after the initial therapy, (2) radiographic evidence of infrabony defects, and (3) a redundant tooth that contains healthy periodontal tissue as a cell source. All patients provided written informed consent according to the GCP. Exclusion criteria included the following: (1) relevant medical conditions contraindicating surgical interventions (e.g., diabetes mellitus; cardiovascular, kidney, liver, or lung disease; or compromised immune system), (2) pregnancy or lactation, and (3) heavy tobacco smoking (more than 11 cigarettes a day). The primary endpoint of this trial was to confirm the safety of autologous PDL cell sheets. This clinical trial finished in 2014, and there were no reported complications in all of the subjects. Observations and recordings of clinical, laboratory, and radiographic outcomes of the patients showed no serious transplant-related adverse event. In the completed cases, reduction of pocket probing depth and clinical attachment gain were observed.

Recent reports have shown that ascorbic acid can assist in the harvesting of PDL cell sheet harvest without the use of temperature-responsive dishes, because of their spontaneous contractions $[11,23,24]$.

\section{Regulation in Japan}

In Japan, two laws, "The Act on the Safety of Regenerative Medicine" and the "Pharmaceuticals, Medical Devices and Other Therapeutic Products Act," came into effect on November $25,2014[25,26]$.

\section{The Act on the Safety of Regenerative Medicine}

This Act covers all kinds of medical technologies using processed cells whose safety and efficacy have not yet been established. All cytotherapies must be registered to the 
Ministry of Health, Labour and Welfare (MHLW) and approved by the special committee based on the potential risk to human health. This Act also stipulates that culturing and processing of cells can be outsourced to the companies whose facilities are inspected by the Pharmaceuticals and Medical Devices Agency (PMDA) to check adherence to the requirements, the usage of GMP-type facilities/equipment, and the quality control requirements for cell processing facilities. Thus, enterprises can participate in the cell processing industry, and they may activate cytotherapeutic regenerative medicine.

\section{Pharmaceuticals, Medical Devices and Other Therapeutic Products Act (PMD Act)}

In this Act, a regenerative medical product is defined as a distinct product from both pharmaceuticals and medical devices, because it is difficult to demand uniform characteristics for regenerative medical products, especially autologous cellular products. Under this Act, regenerative medical products can be approved with conditions and a limited term, but the safety and efficacy of the products need to be confirmed continuously in conjunction with postmarketing safety measures. The approval review process will adopt a 9-month target for regulatory review time, which is consistent with the US Food and Drug Administration's accelerated approval scheme for serious or life-threatening illness.

\section{Conclusion}

Cell Sheet technology is beginning to be used in clinical settings not only for outside of human body but also for inside, because the stability of cell sheets at the transplantation site is relatively high compared to the injection of cells. Although the thickness of cell sheets is less than $50 \mu \mathrm{m}$, multilayering technology of cell sheets may allow production of a sufficient bulk and strength to be of clinical value. In fact, prevascularized tissue has been successfully created in vitro [27, 28]. Thus, indications and clinical trials of cell sheet technology will be increasing based on the governmental promotion in Japan. Although there is a limitation of cell source, embryonic stem cells and induced pluripotent stem cells have a potential to overcome the problem of supply of sufficient cell numbers for regenerative procedures.

\section{Compliance with Ethics Guidelines}

Conflict of Interest All the authors have received grants from the Ministry of Education, Culture, Sports, Science and Technology (MEXT) of Japan; in addition, Dr. Ishikawa has a patent null issued. Dr. Yamato has also received grants from CellSeed Inc., and Dr. Okano has received personal fees as a founder and a member of the board of CellSeed Inc.
Human and Animal Rights and Informed Consent This study was conducted according to the principles expressed in the Declaration ofHelsinki and approved by both the Institutional Review Board of Tokyo Women's Medical University (TWMU) Human Subjects Research and the Japanese Minister of Health, Labour and Welfare in accordance with the "Guidelines on clinical research using human stem cells".

\section{References}

1. Langer R, Vacanti JP. Tissue engineering. Science. 1993;260:920-6.

2. Bartold PM, McCulloch CA, Narayanan AS, Pitaru S. Tissue engineering: a new paradigm for periodontal regeneration based on molecular and cell biology. Periodontol. 2000;24:253-69.

3. Okano T, Yamada N, Sakai H, Sakurai Y. A novel recovery system for cultured cells using plasma-treated polystyrene dishes grafted with poly(N-isopropylacrylamide). J Biomed Mater Res. 1993;27: 1243-51.

4. Nishida K, Yamato M, Hayashida Y, et al. Corneal reconstruction with tissue-engineered cell sheets composed of autologous oral mucosal epithelium. N Engl J Med. 2004;351:1187-96.

5. Burillon C, Huot L, Justin V, et al. Cultured autologous oral mucosal epithelial cell sheet (CAOMECS) transplantation for the treatment of corneal limbal epithelial stem cell deficiency. Invest Ophthalmol Vis Sci. 2012;53:1325-31.

6. Ohki T, Yamato M, Ota M, et al. Prevention of esophageal stricture after endoscopic submucosal dissection using tissue-engineered cell sheets. Gastroenterology. 2012;143:582-8. e1-2.

7. Sawa Y, Yoshikawa Y, Toda K, et al. Safety and efficacy of autologous skeletal myoblast sheets (TCD-51073) for the treatment of severe chronic heart failure due to ischemic heart disease. Circ J. 2015;79:991-9.

8. Sato M, Yamato M, Hamahashi K, Okano T, Mochida J. Articular cartilage regeneration using cell sheet technology. Anat Rec. 2014;297:36-43.

9. Seol YJ, Pellegrini G, Franco LM, Chang PC, Park CH, Giannobile WV. Preclinical methods for the evaluation of periodontal regeneration in vivo. Methods Mol Biol. 2010;666:285-307.

10. Liu Y, Zheng Y, Ding G, et al. Periodontal ligament stem cellmediated treatment for periodontitis in miniature swine. Stem Cells. 2008;26:1065-73.

11. Ding G, Liu Y, Wang W, et al. Allogeneic periodontal ligament stem cell therapy for periodontitis in swine. Stem Cells. 2010;28:1829-38.

12. Hasegawa M, Yamato M, Kikuchi A, Okano T, Ishikawa I. Human periodontal ligament cell sheets can regenerate periodontal ligament tissue in an athymic rat model. Tissue Eng. 2005;11:469-78.

13. Iwata $\mathrm{T}$, Yamato $\mathrm{M}$, Tsuchioka $\mathrm{H}$, et al. Periodontal regeneration with multi-layered periodontal ligament-derived cell sheets in a canine model. Biomaterials. 2009;30:2716-23.

14. Flores MG, Hasegawa M, Yamato M, Takagi R, Okano T, Ishikawa I. Cementum-periodontal ligament complex regeneration using the cell sheet technique. J Periodontal Res. 2008;43:364-71.

15. Flores MG, Yashiro R, Washio K, Yamato M, Okano T, Ishikawa I. Periodontal ligament cell sheet promotes periodontal regeneration in athymic rats. J Clin Periodontol. 2008;35:1066-72.

16. Kim CS, Um YJ, Chai JK, et al. A canine model for histometric evaluation of periodontal regeneration. Periodontology 2000 2011;56:209-26.

17. Seo BM, Miura M, Gronthos S, et al. Investigation of multipotent postnatal stem cells from human periodontal ligament. Lancet. 2004;364:149-55.

18. Tsumanuma Y, Iwata T, Washio K, et al. Comparison of different tissue-derived stem cell sheets for periodontal regeneration in a canine 1-wall defect model. Biomaterials. 2011;32:5819-25. 
19. Kim CS, Choi SH, Chai JK, et al. Periodontal repair in surgically created intrabony defects in dogs: influence of the number of bone walls on healing response. J Periodontol. 2004;75:229-35.

20. Iwata T, Yamato M, Zhang Z, et al. Validation of human periodontal ligament-derived cells as a reliable source for cytotherapeutic use. $\mathrm{J}$ Clin Periodontol. 2010;37:1088-99.

21. Washio K, Iwata T, Mizutani M, et al. Assessment of cell sheets derived from human periodontal ligament cells: a pre-clinical study. Cell Tissue Res. 2010;341:397-404.

22. Yoshida T, Washio K, Iwata T, Okano T, Ishikawa I. Current status and future development of cell transplantation therapy for periodontal tissue regeneration. Int J Dent. 2012;2012:307024.

23. Vaquette C, Fan W, Xiao Y, Hamlet S, Hutmacher DW, Ivanovski S. A biphasic scaffold design combined with cell sheet technology for simultaneous regeneration of alveolar bone/periodontal ligament complex. Biomaterials. 2012;33:5560-73.

24. Feng F, Akiyama K, Liu Y, et al. Utility of PDL progenitors for in vivo tissue regeneration: a report of 3 cases. Oral Dis. 2010;16:20-8.

25. Konomi K, Tobita M, Kimura K, Sato D. New Japanese initiatives on stem cell therapies. Cell Stem Cell. 2015;16:350-5.

26. Hara A, Sato D, Sahara Y. New governmental regulatory system for stem cell-based therapies in Japan. Ther Innov Regul Sci. 2014;48: 681-8.

27. Sakaguchi $\mathrm{K}$, Shimizu $\mathrm{T}$, Horaguchi $\mathrm{S}$, et al. In vitro engineering of vascularized tissue surrogates. Sci Rep. 2013;3:1316.

28. Sekine H, Shimizu T, Sakaguchi K, et al. In vitro fabrication of functional three-dimensional tissues with perfusable blood vessels. Nat Commun. 2013;4:1399. 